【光电工程 / Optoelectronic Engineering】

\title{
异或门自治布尔网络及物理随机数发生器
}

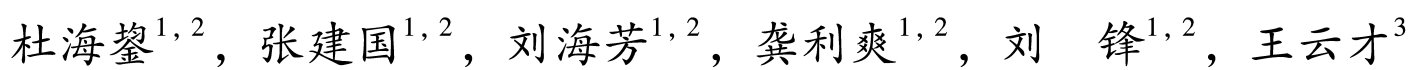

1) 太原理工大学新型传感器与智能控制教育部和山西省重点实验室, 山西太原 030024 ;

2) 太原理工大学物理与光电工程学院, 山西太原 030024 ；3) 广东工业大学信息工程学院, 广东广州 510006

摘 要：自治布尔网络作为可调控的非线性系统，易于产生混沌信号. 利用二输入异或门的非线性特 性实现一种可产生高摘值混沌的 18 节点自治布尔网络电路. 通过引入滤波系数, 建立该电路的改进数学模 型. 通过数值仿真和电路对比实验研究滤波系数和时延参数对网络动态特性的影响. 数值仿真结果与电路 实验现象基本一致. 当节点之间传输延迟不相等时，滤波系数对混沌的产生具有调控作用，表明引入滤波 系数后的数学模型可以更客观描述电路中的物理现象. 借助分岔图、李雅普诺夫指数及排序摘进一步分析 自治布尔网络电路的非线性特性和随机性，表明该电路输出的混沌序列具有高带宽和高摘值的特点. 将该 电路用于产生高速物理随机序列, 并通过了美国国家标准与技术研究院 NIST SP 800-22 随机性检测标准.

关键词：物理电子学；非线性系统；布尔网络；混沌电路；物理随机数；保密通信

中图分类号：TN918 文献标志码：A doi：10.3724/SP. J. 1249.2021.01103

\section{Autonomous Boolean network and physical random number generator based on XOR gate}

\section{DU Haijun ${ }^{1,2}$, ZHANG Jianguo ${ }^{1,2}$, LIU Haifang ${ }^{1,2}$, GONG Lishuang ${ }^{1,2}$, LIU Feng ${ }^{1,2}$, and WANG Yuncai ${ }^{3}$}

1) Key Laboratory of Advanced Transducers and Intelligent Control System of Ministry of Education and Shanxi Province, Taiyuan University of Technology, Taiyuan 030024, Shanxi Province, P. R. China

2) College of Physics and Optoelectronics, Taiyuan University of Technology, Taiyuan 030024, Shanxi Province,P. R. China 3) College of Information and Engineering, Guangdong University of Technology, Guangzhou 510006, Guangdong Province,P. R. China

\begin{abstract}
As a controllable nonlinear system, autonomous Boolean network which is easy to generate chaotic signals has become a hot research topic. We realize an 18-node autonomous Boolean network circuit which can generate high entropy chaos by using the nonlinear characteristics of two-input XOR gate, and establish an improved mathematical model for the circuit by introducing the filter coefficient. Through numerical simulation and circuit comparison experiment, we study the influence of filter coefficient and delay parameters on network dynamic characteristics of this model. The numerical simulation results are basically consistent with those of the experimental phenomena. When the transmission delay between nodes is not equal, the filter coefficient has a modulation effect on the generation of chaos, which well indicates that the introduction of the filter coefficient makes the improved mathematical model more objective to describe the physical phenomena in the circuit. In addition, using bifurcation diagram, Lyapunov exponent and permutation entropy, we further analyze the nonlinear characteristics and

Received: 2020-03-05; Accepted: 2020-04-05

Foundation: National Natural Science Foundation of China (61671316); Natural Science Foundation of Shanxi Province (201801D121145)

Corresponding author: Associate professor ZHANG Jianguo. E-mail:zhangjianguo@ tyut.edu.cn

Citation: DU Haijun, ZHANG Jianguo, LIU Haifang, et al. Autonomous Boolean network and physical random number generator based on XOR gate $[\mathrm{J}]$. Journal of Shenzhen University Science and Engineering, 2021, 38(1): 103-109. (in Chinese)
\end{abstract}


randomness of the autonomous Boolean network. The output chaotic sequence of the network has the characteristics of high bandwidth, high entropy and strong robustness, which is used to generate high speed physical random sequences and has passed the NIST SP 800-22 randomness tests of National Institute of Standards and Technology of America.

Key words : physical electronics; nonlinear system; Boolean network; chaotic circuit; physical random number; secure communications

自治布尔网络 ( autonomous Boolean network, $\mathrm{ABN}$ ) 是与离散映射布尔网络相对应的连续动力学 系统 $^{[1]}$ ，具有时间、空间及状态连续性. 与同步布 尔网络 (synchronous Boolean network, SBN) 相比 ${ }^{[2]}$, $\mathrm{ABN}$ 的相空间从有限维扩展到无限维, 使其能够产 生更复杂的动力学行为. 作为典型的非线性动力学 系统, $\mathrm{ABN}$ 可以呈现周期振荡、分岔及混沌等非线 性状态. 混沌具有对初值敏感、随机及不可预测等 特性, 在密码学和物理随机数产生等领域极具应用 前景 ${ }^{[3-5]}$, 激发了广泛的研究兴趣.

ROSIN 等 ${ }^{[6]}$ 首次提出基于三输人异或门结构的 16 节点 $\mathrm{ABN}$, 并在现场可编程门阵列 ( field programmable gate array, FPGA) 平台产生单通道速率为 $100 \mathrm{Mbit} / \mathrm{s}$ 、并行速率为 $12.8 \mathrm{Gbit} / \mathrm{s}$ 的物理随机数. 在这项开创性研究的推动下, ROSIN 等 ${ }^{[7-8]}$ 研究了 $\mathrm{ABN}$ 的受迫和集群同步等问题. 马荔等 ${ }^{[9-11]}$ 提出不 同拓扑结构的 $\mathrm{ABN}$ ，并利用香农熵、自相关性、最 大李雅普诺夫指数及记忆时间等指标分析混沌的随 机性和不可预测性. DONG 等 ${ }^{[10]}$ 研究时延参数对 $\mathrm{ABN}$ 的影响, 指出时延参数的差异性是控制 $\mathrm{ABN}$ 产生混沌的关键. ROSIN 等 ${ }^{[12-13]}$ 则进一步指出, $\mathrm{ABN}$ 自身存在不稳定机制，在一个拓扑结构固定的 网络中，当节点数量超过阈值时，网络会出现从周 期态或稳态到混沌态的相变. 与实验研究同步开展 的还有 $\mathrm{ABN}$ 的理论建模工作, 目前可用于 $\mathrm{ABN}$ 建 模的数学方法主要有 GHIL 等 ${ }^{[14-15]}$ 提出的布尔延迟 方程 ( Boolean delay equations, BDEs) 和 GLASS 等 ${ }^{[16-18]}$ 提出的分段线性微分方程 (piecewise-linear differential equations, PDEs). 相比于使用 BDEs 方 程建模 ${ }^{[19]}$ ，基于 PDEs 方程建立的 $\mathrm{ABN}$ 模型可以 较准确刻画 $\mathrm{ABN}$ 电路中的混沌现象 ${ }^{[6]}$ ，但该模型 同样存在不足，如模型未考虑节点的低通滤波效应 对 $\mathrm{ABN}$ 的影响, 不能合理解释 $\mathrm{ABN}$ 电路实验中出 现的周期态到混沌态的相变等物理现象.

针对上述问题，本研究通过理论与实验研究一 种基于二输人异或门的 18 节点 ABN. 基于 PDEs, 通过引人滤波系数建立该 $\mathrm{ABN}$ 的改进数学模型,
仿真分析该 $\mathrm{ABN}$ 在不同时延参数和滤波系数情况 下的工作状态 (周期态和混沌态), 证实滤波系数具 有抑制和控制混沌产生的作用. 采用通用的电子异 或门器件实现 $\mathrm{ABN}$ 电路, 实验结果与改进数学模 型的数值仿真结果基本一致, $\mathrm{ABN}$ 电路中存在的 “混沌-周期-混沌” 2 次相变物理现象, 可以从改进 的 ABN 数学模型中得到合理解释. 实验还发现该 $\mathrm{ABN}$ 电路产生的混沌信号具有良好的随机性、鲁棒

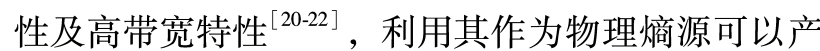
生实时速率达 $100 \mathrm{Mbit} / \mathrm{s}$ 的高速物理随机数序列, 美国国家标准与技术研究院随机性检测结果表明, 所产生随机数具有良好的随机统计特性.

\section{1 自治布尔网络}

本研究设计的自治布尔网络如图 1 , 其由 18 个 节点构成, 每个节点有两路输人和两路输出信号, 输人信号分别来自左右两个相邻节点, 输出信号也 分别传输至这两个节点. 在该 $\mathrm{ABN}$ 中，除节点 1 执 行异或非运算 (XNOR) 外，其余 17 个节点（2 18）均执行异或运算 (XOR).

使用分段线性微分方程建立该 $\mathrm{ABN}$ 网络的数 学模型为

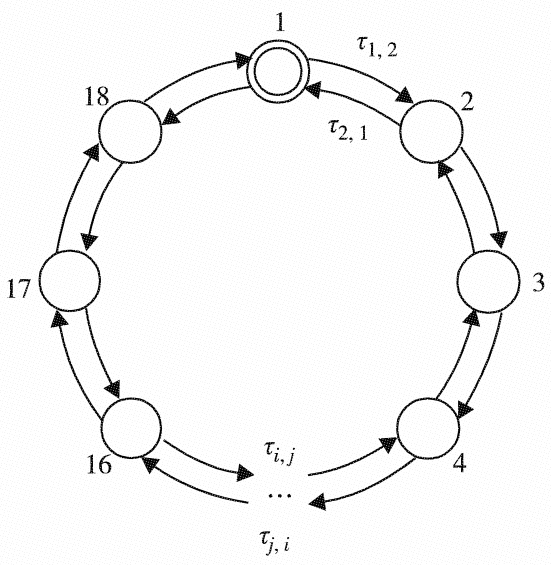

图 1 布尔网络示意图

Fig. 1 Autonomous Boolean network structure 


$$
\begin{gathered}
\left\{\begin{array}{c}
\tau_{\mathrm{LP}} \frac{\mathrm{d} x_{1}}{\mathrm{~d} t}=-x_{1}(t)+X_{2}\left(t-\tau_{1,2}\right) \oplus \\
X_{18}\left(t-\tau_{1,18}\right) \oplus 1 \\
\tau_{\mathrm{LP}} \frac{\mathrm{d} x_{2}}{\mathrm{~d} t}=-x_{2}(t)+X_{3}\left(t-\tau_{2,3}\right) \oplus \\
X_{1}\left(t-\tau_{2,1}\right) \\
\vdots \\
\tau_{\mathrm{LP}} \frac{\mathrm{d} x_{18}}{\mathrm{~d} t}=-x_{18}(t)+X_{1}\left(t-\tau_{18,1}\right) \oplus \\
X_{17}\left(t-\tau_{18,17}\right)
\end{array}\right. \\
X_{i}(t)= \begin{cases}1, & x_{i}(t)>x_{\mathrm{th}} \\
0, & x_{i}(t) \leqslant x_{\mathrm{th}}\end{cases}
\end{gathered}
$$

其中, $x_{i} \in B=\{0,1\}(i=1,2, \cdots, 18)$ 是第 $i$ 个节 点的状态变量; $X_{i}$ 为节点 $i$ 的输出; $x_{\mathrm{th}}$ 为节点阈值 (一般取值为 0.5$) ; \tau_{i, j}(i, j=1,2, \cdots, 18)$ 为信号 从节点 $j$ 到节点 $i$ 的传输延迟; $\tau_{\mathrm{LP}}$ 为滤波系数; $\oplus$ 表 示逻辑异或 $(\mathrm{XOR})$ 运算.

\section{$2 \mathrm{ABN}$ 数值仿真}

利用 4 阶 Runge-Kytta 方法 ${ }^{[23]}$ 对系统方程 (1) 进行数值求解, 研究当参数 $\tau_{i, j}$ 和 $\tau_{\mathrm{LP}}$ 取不同值时,
$\mathrm{ABN}$ 的动态变化.

当 $\tau_{i, j}$ 均相等时, 令 $\tau_{i, j}=T(T$ 为常数 $)$, 然后 连续改变 $\tau_{\mathrm{LP}}$ 的取值. 仿真结果表明, 当所有传输 延迟 $\tau_{i, j}$ 都相等时, 无不动点的 $\mathrm{ABN}$ 网络将处于周 期振荡状态.

当 $\tau_{i, j}$ 不相等时, 令 $\tau_{i, j}=T \pm \varepsilon$ ( $\varepsilon$ 为误差 $)$, 然后连续改变 $\tau_{\mathrm{LP}}$ 的取值. 仿真结果表明, 若忽略 方程参数 $\tau_{\mathrm{LP}}$, 则无不动点的 $\mathrm{ABN}$ 网络将总是处于 混沌状态; 当引人参数 $\tau_{\mathrm{LP}}$, 则无不动点的 $\mathrm{ABN}$ 网 络将处于混沌或周期状态，表明滤波系数 $\tau_{\mathrm{LP}}$ 对混 沌的产生具有调控或抑制作用. 图 2 为 $\tau_{\mathrm{LP}}$ 分别取 $2.4 、 3.9$ 及 $4.3 \mathrm{~ns} 、 \tau_{i, j}=2.7 \mathrm{~ns}$ 及 $\varepsilon=0.5 \mathrm{~ns}$ 时, $\mathrm{ABN}$ 产生的时序和相图.

最大李雅普诺夫指数是衡量非线性系统动力学

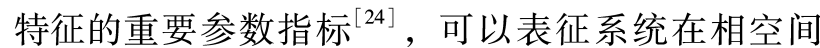
中相邻轨道收玫或发散的平均指数率，当最大李雅 普诺夫指数值小于等于 0 时，系统为周期状态; 大 于 0 时为混沌状态; 无穷大时为噪声状态. 本研究 利用轨道跟踪法中的小数据法 ${ }^{[25]}$, 计算得到图 2 中时间序列的最大李雅普诺夫指数 (the largest Lyapunov exponent) 分别为 $L_{1}=0.32 \mathrm{~ns}^{-1} 、 L_{2}=$ $0 \mathrm{~ns}^{-1}$ 及 $L_{3}=0.26 \mathrm{~ns}^{-1}$.

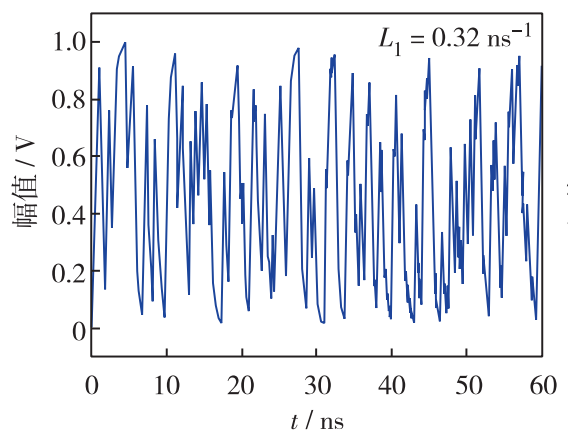

(a) $\tau_{\mathrm{LP}}=2.4 \mathrm{~ns}$ 时的时序

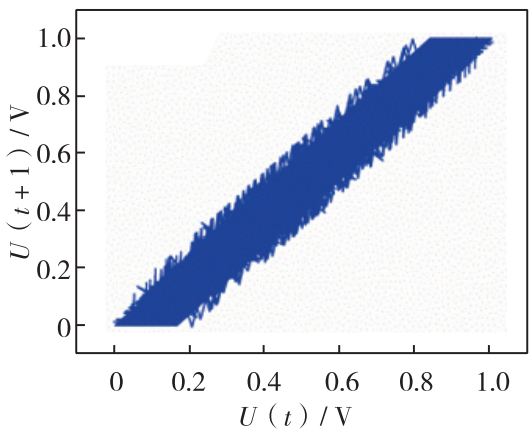

(d) $\tau_{\mathrm{LP}}=2.4 \mathrm{~ns}$ 时的相图

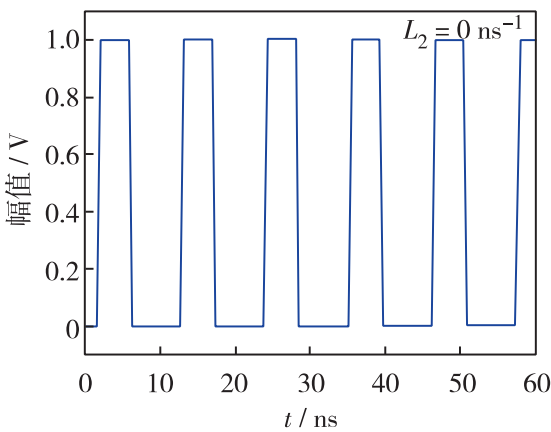

(b) $\tau_{\mathrm{LP}}=3.9 \mathrm{~ns}$ 时的时序

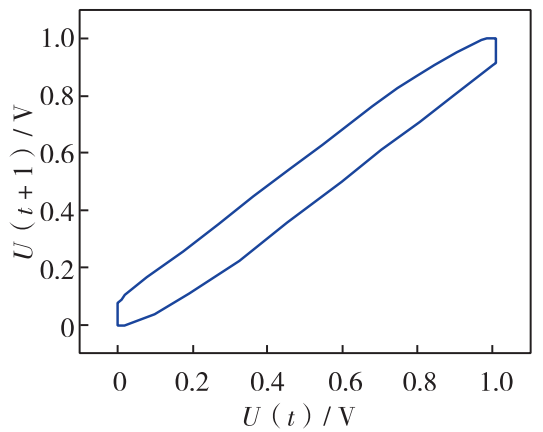

(e) $\tau_{\mathrm{LP}}=3.9 \mathrm{~ns}$ 时的相图

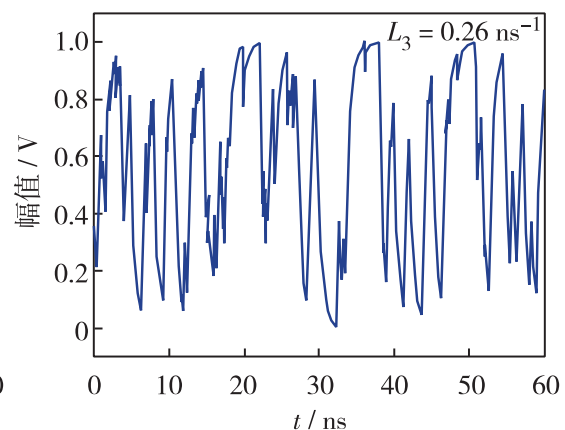

(c) $\tau_{\mathrm{LP}}=4.3 \mathrm{~ns}$ 时的时序

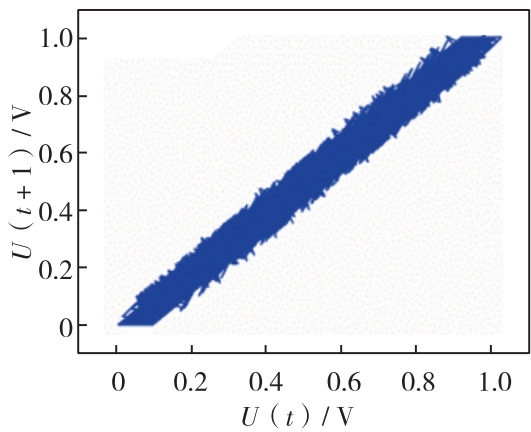

(f) $\tau_{\mathrm{LP}}=4.3 \mathrm{~ns}$ 时的相图

图 2 自治布尔网络输出时序和相图

Fig. 2 (Color online) Autonomous Boolean network output time series and phase diagrams 


\section{$3 \mathrm{ABN}$ 电路实验}

$\mathrm{ABN}$ 电路实验装置如图 3，电路中的节点由德 州仪器公司的 74AUC1G86 异或门芯片构成, 其工 作电压为 $0.8 \sim 2.7 \mathrm{~V} . \mathrm{ABN}$ 电路中的节点间传输延 迟可以表示为 $\tau_{i, j}=t_{\mathrm{pd}}+t_{\text {link }}\left(t_{\mathrm{pd}}\right.$ 为异或门传输延迟

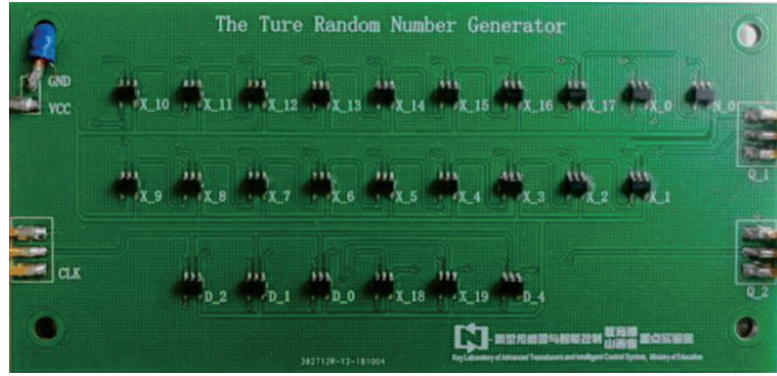

图 3 布尔网络电路实物图

Fig. 3 (Color online) Prototype board of Boolean circuit
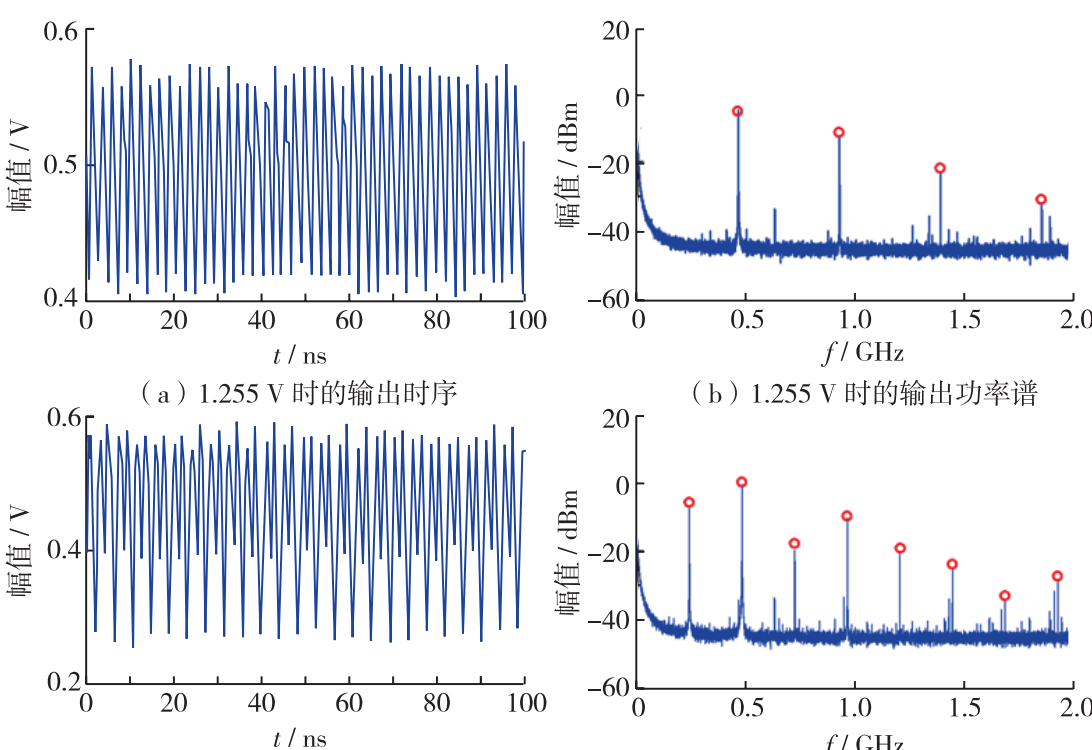

(d) $1.310 \mathrm{~V}$ 时的输出时序

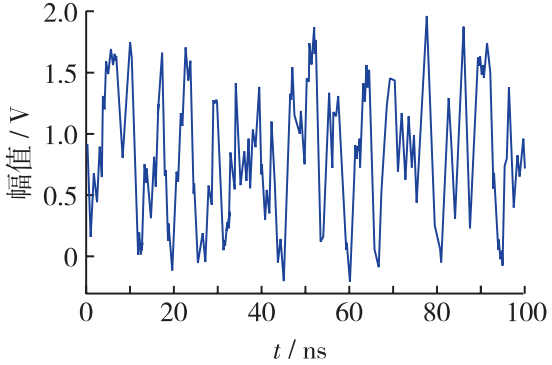

( g ) $1.800 \mathrm{~V}$ 时的输出时序

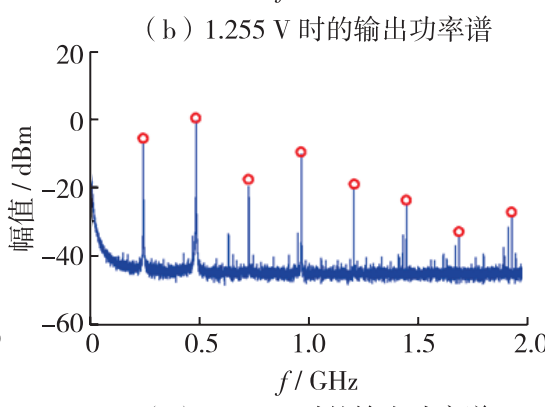

(e) $1.310 \mathrm{~V}$ 时的输出功率谱

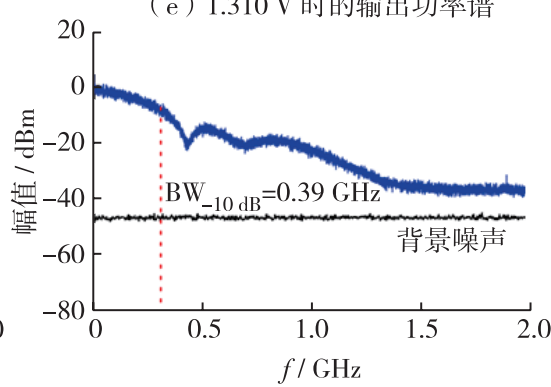

(h) $1.800 \mathrm{~V}$ 时的输出功率谱
时间; $t_{\text {link }}$ 为信号在导线上的传输延迟), 由于 $t_{\mathrm{pd}} \gg$ $t_{\text {link }}$, 有 $\tau_{i, j} \approx t_{\mathrm{pd}}$. 由芯片手册可知, $t_{\mathrm{pd}}$ 的工作电压 越低, $t_{\mathrm{pd}}$ 值越大. 根据该特性, 实验中可以通过改 变电路的供电电压达到调谐节点信号传输延迟 $\tau_{i, j}$ 的目的. 此外, 虽然异或门采用相同半导体工艺制 造, 但是制造中的微小误差无法避免, 因此, 在 $\mathrm{ABN}$ 电路中, 节点间的传输延迟 $\tau_{i, j}$ 总是不相等的.

图 4 为供电电压为 $1.255 、 1.310$ 和 $1.800 \mathrm{~V}$ 时, $\mathrm{ABN}$ 电路输出信号（节点 2 输出）的时序、 功率谱和相图. 可见, 当供电电压为 $1.255 \mathrm{~V}$ 时, $\mathrm{ABN}$ 电路的输出信号呈单倍周期振荡, 其基频频率 为 $459 \mathrm{MHz}$; 当供电电压逐渐升高至 $1.310 \mathrm{~V}$ 时, 相图呈现出二倍周期，基频频率为 $238 \mathrm{MHz}$; 进一 步调节供电电压至 $1.800 \mathrm{~V}$ 时, $\mathrm{ABN}$ 电路输出信号 随时间的演化变得复杂、无规则，相图出现吸引 子, 其功率谱由离散谱转变为连续谱, 这是混沌信 号的典型特征; 该信号还具有宽频带特征，其 -10 dB 带宽可达 $390 \mathrm{MHz}$.

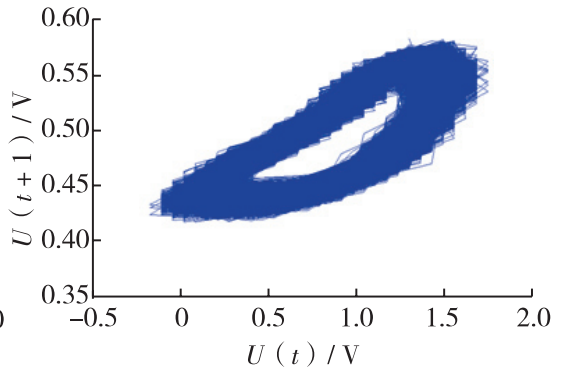

(c) $1.255 \mathrm{~V}$ 时的输出相图

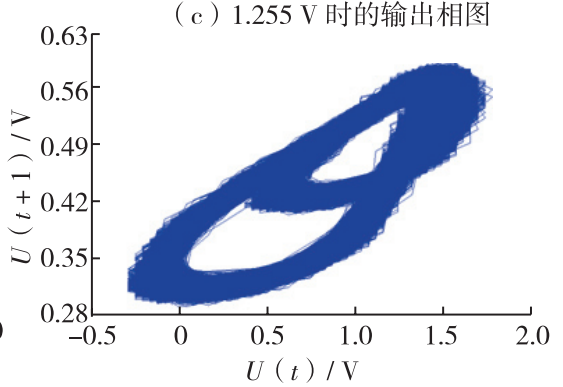

(f) $1.310 \mathrm{~V}$ 时的输出相图

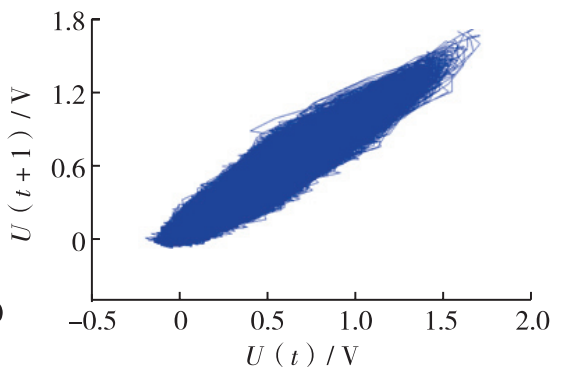

(i) $1.800 \mathrm{~V}$ 时的输出相图

图 4 不同供电电压时的布尔电路输出信号时序、功率谱及相图

Fig. 4 (Color online) Time series, power spectra and phase diagrams of the output signals of Boolean circuit with different supply voltages 
根据文献 $[18]$ 方法, 分别以 $\mathrm{ABN}$ 电路供电电 压和结点 2 输出信号的相邻两个上升沿之间的时间 隔为参数, 绘制混沌时序分岔图, 如图 5 (a). 其 中, 点带状区域（如区域 $\mathrm{a}$ 和 $\mathrm{d}$ ) 表示电路进入混 沌振荡状态，周期振荡窗口（如区域 $b$ 和 $\mathrm{c}$ ）则分 布其间. ABN 电路中低通滤波效应的作用导致周期 状态的小范围出现，该现象也与数值仿真结果相互 印证, 表明 $\mathrm{ABN}$ 数学模型中引入滤波系数的合理 性. 此外, 宽阔的点带状区域 $\mathrm{d}$ 表明该 $\mathrm{ABN}$ 在较大 的供电电压参数范围 $(1.4 \sim 2.7 \mathrm{~V})$ 内可以稳定工 作于混沌振荡状态, 具有较强鲁棒性.
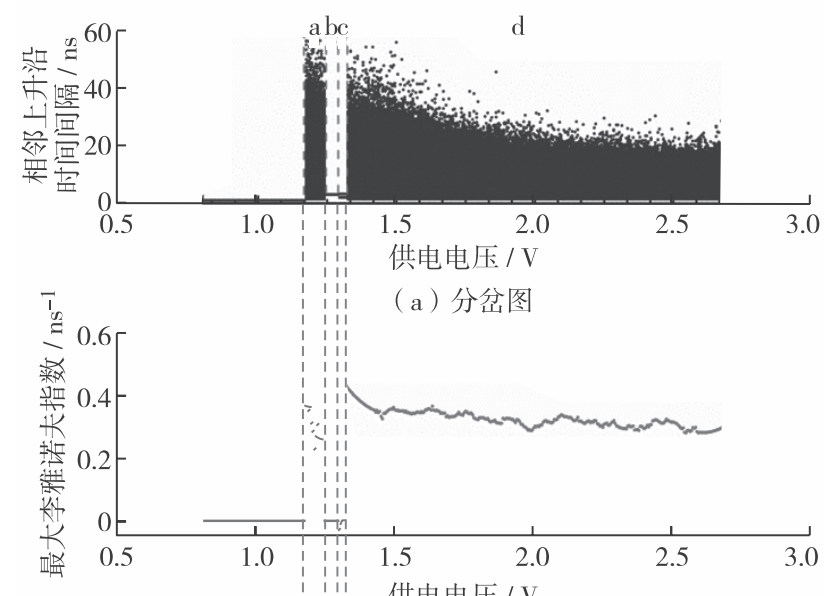

(b) LLE

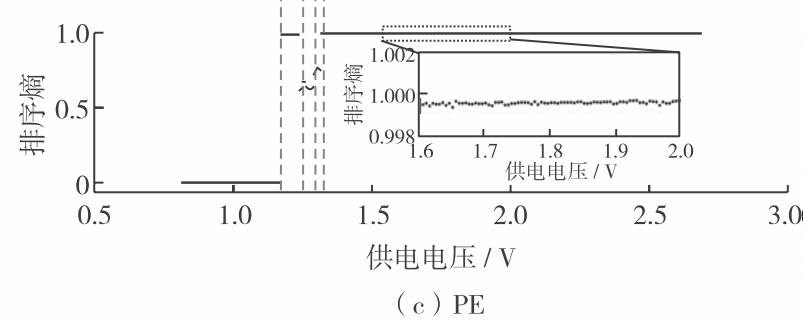

图 5 布尔电路输出信号随供电电压增加的变化趋势

Fig. 5 Output signals of the Boolean circuit as function of the supply voltage

排序熵 (permutation entropy, PE) 是度量混沌信 号随机性的重要指标 ${ }^{[21]}$, 定义为

$$
h(p)=-\sum_{i=1}^{M !} p_{i}(\pi) \operatorname{lb} p_{i}(\pi)
$$

其中, $h(p)$ 为排序熵; $\pi$ 为符号序列 $; p_{i}(\pi)$ 为 $\pi$ 相 对出现频率; $M$ 为嵌人维度. 对式 (2) 归一化可得

$$
H(p)=\frac{h(p)}{\operatorname{lb}(M !)}
$$

对于给定时间序列 $\left\{x_{1}, x_{2}, \cdots, x_{i}, \cdots, x_{N}\right\}, N$ 为 数据长度，使用 TAKENS 定理将其相空间重构为
$S=\left[x_{t}, x_{t+l}, \cdots, x_{t+(M-1) l}\right]$, 其中, $l$ 为时间延迟. 对 重构后的序列递增排序可得 $s=\left[x_{t+\left(r_{1}-1\right) l}, x_{t+\left(r_{2}-1\right) l}\right.$, $\left.\cdots, x_{t+\left(r_{M}-1\right) l}\right]$. 式 (2) 中的 $\pi$ 为该序列被映射为所有 $M !$ 种排列中的唯一顺序模式，即 $\pi=\left(r_{1}, r_{2}, \cdots\right.$, $\left.r_{M}\right), r_{1}, r_{2}, \cdots, r_{M}$ 代表重构分量中每个元素的序号. 式(2) 中的 $\pi$ 为符号序列，则 $\pi$ 在时间序列分析中 出现的次数可以用 $f(\pi)$ 表示, 对应概率为

$$
p_{i}(\pi)=f(\pi) /(N-(M-1) \tau)
$$

实验中取 $M=5, l=30$. 图 $5(\mathrm{c})$ 为 $\mathrm{ABN}$ 电路 在不同供电电压下的排序熵测量结果. 当电路处于 混沌状态时, PE 的值始终接近于 1, 表明 ABN 电 路在较大的供电电压参数范围内可以输出的混沌信 号具有良好的随机性.

\section{4 基于 $\mathrm{ABN}$ 的物理随机数发生器}

ABN 电路输出的混沌序列具有高带宽、高熵值 以及较强鲁棒性的特点, 可作为物理随机数发生器 (physical-RNG) 的熵源. 本研究设计的 physical-RNG 如图 6, 分为 $\mathrm{ABN}$ 熵源和熵提取器 2 部分. 熵提取 器由 1 个 3 输人 XOR 门和 4 个 D 触发器构成, 其 中, 利用 3 个 $\mathrm{D}$ 触发器实现对 $\mathrm{ABN}$ 熵源输出混沌 信号（节点 1 、节点 7 和节点 13 ) 的采样和量化, 产生 $0 、 1$ 比特随机序列. 而后随机序列再经 3 路异 或和 D 触发器波形整形, 进一步降低序列中的偏差 和相关性, 最终产生高质量的物理随机数序列.
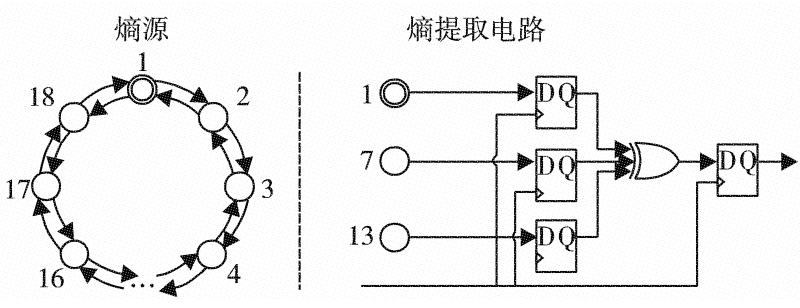

图 6 物理随机数发生器

Fig. 6 Physical random number generator

实验中将 $\mathrm{D}$ 触发器的时钟设定为 $100 \mathrm{MHz}$, 此 时该 physical-RNG 实时输出速率为 $100 \mathrm{Mbit} / \mathrm{s}$ 的随 机数序列. 当 $\mathrm{ABN}$ 电路供电电压为 $1.8 \mathrm{~V}$ 时, $\tau_{i, j}=$ $1 \mathrm{~ns}, \varepsilon=0.4 \mathrm{~ns}$, 采集 $1 \mathrm{Gbit}$ 随机数 $(1000$ 组, 每 组 1 Mbit) 进行美国国家标准与技术研究院 NIST SP 800-22 规定的 15 项测试，显著水平 $\alpha$ 设置为 0.01 , 测试结果包括 $p$ 值及 proportion, 当 $p \geqslant$ 0.0001 , proportion $\geqslant 0.9806$ 时, 表示通过. 测试 结果如图 7 , 可见, 产生的随机数能通过全部测试. 


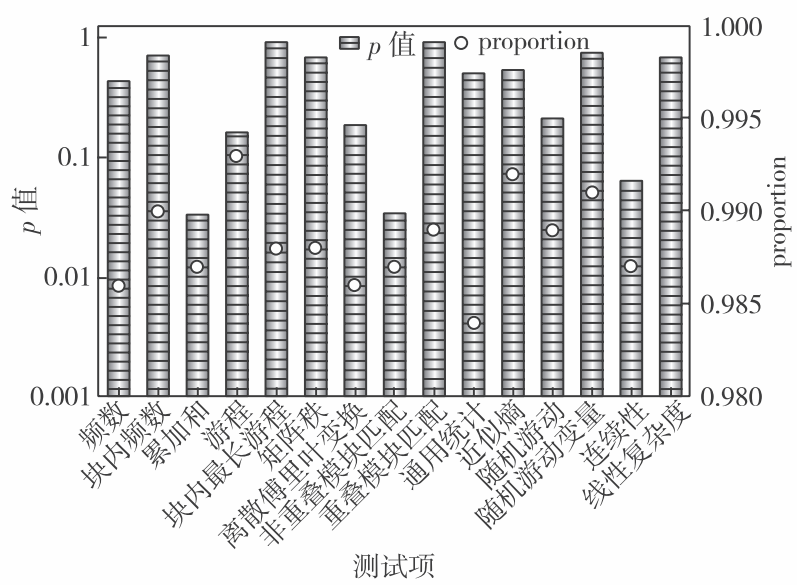

图 7 NIST 测试结果

Fig. 7 Typical results of NIST statistical test

表 1 是基于 $\mathrm{ABN}$ 网络的物理随机数发生器与 文献 $[6,26-28]$ 的对比结果. 可见, 本研究提出的 物理随机数发生器具有速率高和每比特消耗能量较 低的特点.

\section{表 1 本研究 physical-RNG 与其他文献中 随机数发生器的性能比较}

Table 1 Comparison of the proposed physical-RNG with other integrated RNGs in the literature

\begin{tabular}{|c|c|c|c|c|}
\hline $\begin{array}{l}\text { 随机数 } \\
\text { 发生器 }\end{array}$ & 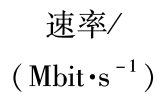 & $\begin{array}{c}\text { 功耗/ } \\
\mathrm{mW}\end{array}$ & $\begin{array}{l}\text { 每 bit 所需能 } \\
\text { 量 } /\left(\mathrm{nJ} \cdot \mathrm{bit}^{-1}\right)\end{array}$ & 熵 源 \\
\hline 文献 [26] & 1.40 & 3.9 & 2.780 & 热噪声 \\
\hline 文献 [27] & 0.04 & 1.0 & 25.000 & 亚稳态 \\
\hline 文献 [28 ] & 10.00 & 29.0 & 2.900 & 离散混沌系统 \\
\hline 文献 $[6]$ & 100.00 & & & $\begin{array}{l}\text { 连续混沌系统 } \\
\text { (三输人 } \mathrm{ABN})\end{array}$ \\
\hline 本研究 & 100.00 & 76.8 & 0.768 & $\begin{array}{l}\text { 连续混沌系统 } \\
\text { (二输人 } \mathrm{ABN} \text { ) }\end{array}$ \\
\hline
\end{tabular}

\section{结 语}

本文研究一种二输人异或门的 18 节点 $\mathrm{ABN}$, 在 PDEs 基础上引人滤波系数建立该 ABN 的改进数 学模型, 利用 4 阶 Runge-Kytta 方法对模型进行求 解. 当改变时延参数和滤波系数时, $\mathrm{ABN}$ 会呈现周 期态和混沌态，表明滤波系数具有抑制和控制混沌 产生的作用.

实验采用电子器件构建仿真模型, 通过改变电 路的供电电压调节节点之间的传输延迟，从绘制的
分岔图中可以观察到电路的混沌和周期状态，与仿 真结果一致. 李雅普诺夫指数和排序熵随电压变化 的演变过程表明该网络在宽幅工作电压范围内均可 以输出随机性良好的混沌信号，因此，使用 100 $\mathrm{MHz}$ 高频时钟信号对其进行量化采样，高速物理随 机数生成速率 $100 \mathrm{Mbit} / \mathrm{s}$, 国际行业标准 NIST SP 800-22 测试结果表明，获得的随机比特序列具 有良好的统计学特性. 以上仿真和实验研究完善了 自治布尔网络熵源结构的理论分析，为混沌物理随 机数发生器的深入研究提供参考.

\footnotetext{
基金项目：国家自然科学基金资助项目（61671316); 山西省自然 科学基金资助项目 (201801D121145)

作者简介：杜海挤（1993-)，太原理工大学硕士研究生．研究方 向: 物理随机数芯片安全性分析. E-mail: duhj68@ 163.com

引文：杜海栓，张建国，刘海芳，等。异或门自治布尔网络及 物理随机数发生器 $[\mathrm{J}]$. 深圳大学学报理工版, 2021, 38 (1) : 103-109.
}

\section{参考文献／References:}

[ 1 ] MESTL T, BAGLEY R J, GLASS L. Common chaos in arbitrarily complex feedback networks [ J ]. Physical Review Letters, 1997, 79(4) : 653-656.

[ 2 ] GREIL F, DROSSEL B. Dynamics of critical Kauffman networks under asynchronous stochastic update $[\mathrm{J}]$. Physical Review Letters, 2005, 95(4) : 048701

[ 3 ] 袁泽世, 李洪涛, 朱晓华. 基于忆阻器的数模混合随机 数发生器 $[\mathrm{J}]$. 物理学报, 2015, 64(24): 87-96.

YUAN Zeshi, LI Hongtao, ZHU Xiaohua. A digital-analog hybrid random number generator based on memristor $[\mathrm{J}]$. Acta Physica Sinica, 2015, 64(24) : 87-96. (in Chinese)

[ 4 ] 党小宇, 李洪涛, 袁泽世, 等. 基于数模混合的混沌映 射实现 $[J]$. 物理学报, 2015, 64(16): 171-179.

DANG Xiaoyu, LI Hongtao, YUAN Zeshi, et al. Chaotic map implementation based on digital-analog hybrid method [J]. Acta Physica Sinica, 2015, 64(16) : 171-179. (in Chinese)

[ 5 ] 李 璞, 江 镭, 孙媛媛, 等. 面向全光物理随机数发 生器的混沌实时光采样研究 $[\mathrm{J}]$. 物理学报, 2015,64 (23) : 25-34.

LI Pu, JIANG Lei, SUN Yuanyuan, et al. Study on realtime optical sampling of chaotic laser for all-optical physical random number generator $[\mathrm{J}]$. Acta Physica Sinica, 2015, 64(23) : 25-34. (in Chinese)

[ 6 ] ROSIN D P, RONTANI D, GAUTHIER D J. Ultra-fast generation of true random numbers using hybrid Boolean networks $[\mathrm{J}]$. Physical Review E: Statistical Nonlinear \& 
Soft Matter Physics, 2012, 87(4): 1316-1326.

[ 7 ] ROSIN D P, RONTANI D, GAUTHIER D J, et al. Control of synchronization patterns in neural-like Boolean networks [J]. Physical Review Letters, 2013, 110(10): 104102.

[ 8 ] RIVERA D, CAMPOS C E, CAMPOS C I, et al. Forced synchronization of autonomous dynamical Boolean networks [J]. Chaos, 2015, 25(8): 083113.

[ 9 ] 马 荔, 张建国, 李 璞, 等. 基于自治布尔网络的高 速物理随机数发生器研究 $[\mathrm{J}]$. 中南大学学报自然科 学版, 2018, 284(4): 124-128.

MA Li, ZHANG Jianguo, LI Pu, et al. High-speed physical random number generator based on autonomous Boolean networks $[\mathrm{J}]$. Journal of Central South University Science and Technology, 2018, 284(4): 124-128. (in Chinese)

[10] DONG Lihua, YANG Hui, ZENG Yong. Analysis and improvement of true random number generator based on autonomous Boolean network $[\mathrm{C}] / /$ The 13th International Conference on Computational Intelligence and Security ( CIS). Hong Kong, China: IEEE, 2017: 243-247.

[11] 龚利爽, 侯二林, 刘海芳, 等. 布尔混沌系统的物理随 机性分析 $[J]$. 通信学报, 2019, 40(3) : 190-195.

GONG Lishuang, HOU Erlin, LIU Haifang, et al. Physical random analysis of Boolean chaos $[\mathrm{J}]$. Journal on Communications, 2019, 40(3) : 190-195. (in Chinese)

[12] ROSIN D P, RONTANI D, GAUTHIER D J. Emergence of dynamical complexity in autonomous Boolean networks [EB/OL]. arXiv, 2012 [2020-01-05]. https://arxiv. org/abs/1209. 0142v1.

[13] 张琪琪, 张建国, 李 璞, 等. 基于布尔混沌的物理随 机数发生器 $[\mathrm{J}]$. 通信学报, 2019, 40(1) : 201-206.

ZHANG Qiqi, ZHANG Jianguo, LI Pu, et al. Booleanchaos-based physical random number generator $[\mathrm{J}]$. Journal on Communications, 2019, 40(1) : 201-206. (in Chinese)

[14] GHIL M, MULLHAUPT A. Boolean delay equations II. periodic and aperiodic solutions $[\mathrm{J}]$. Journal of Statistical Physics, 1985, 41(1/2) : 125-173.

[15] CHENG X, SUN M, SOCOLAR J E S. Autonomous Boolean modelling of developmental gene regulatory networks $[\mathrm{J}]$. Journal of the Royal Society Interface, 2012, 10(78) : 20120574

[16] GLASS L, HILL C. Ordered and disordered dynamics in random networks [J]. EPL, 2007, 41(6) : 599-604.

[17] EDWARDS R, GLASS L. Combinatorial explosion in model gene networks $[\mathrm{J}]$. Chaos, $2000,10(3)$ : 691704.

[18 ] MESTL T, BAGLEY R J, GLASS L. Common chaos in arbitrarily complex feedback networks [J]. Physical Review Letters, 1997, 79(4) : 653-656.

[19] ZHANG R, HL D S C, GAO Z, et al. Boolean chaos [J]. Physical Review E, 2009, 80(2) : 045202.

[20 ] IMMOREEV I, TAO T H. UWB radar for patient monitoring $[\mathrm{J}]$. IEEE Aerospace \& Electronic Systems Magazine, 2008, 23(11) : 11-18.

[21] BANDT C, POMPE B. Permutation entropy: a natural complexity measure for time series $[\mathrm{J}]$. Physical Review Letters, 2002, 88(17): 174102.

[22] AIS 31. Functionality classes and evaluation methodology for true (physical) random number generators $[S]$.

[23] KLOEDEN P E, PEARSON R A. The numerical solution of stochastic differential equations $[\mathrm{J}]$. The ANZIAM Journal, 1977, 20(1) : 8-12.

[24] GAO J B, HU J, TUNG W W, et al. Distinguishing chaos from noise by scale-dependent Lyapunov exponent [J]. Physical Review E, 2006, 74(6) : 066204.

[25 ] ROSENSTEIN M T, COLLINS J J, LUCA C J D. A practical method for calculating largest Lyapunov exponents from small data set $[\mathrm{J}]$. Physica D: Nonlinear Phenomena, $1993,65(1 / 2): 117-134$.

[26] BUCCI M, GERMANI L, LUZZI R, et al. A high-speed oscillator-based truly random number source for cryptographic applications on a smart card IC $[\mathrm{J}]$. IEEE Transactions on Computers, 2003, 52(4) : 403-409.

[27 ] KINNIMENT D J, CHESTER E G. Design of an on-chip random number generator using metastability $[\mathrm{C}] / /$ Proceedings of the 28th European Solid-State Circuits Conference. Florence, Italy: IEEE, 2002 : 595-598.

[28] PARESCHI F, SETTI G, ROVATTI R. Implementation and testing of high-speed CMOS true random number generators based on chaotic systems $[\mathrm{J}]$. IEEE Transactions on Circuits and Systems I: Regular Papers, 2010, 57 (12) : 3124-3137.

【中文责编：方 圆; 英文责编：溯 心】 